Projets

de paysage

\section{Projets de paysage}

Revue scientifique sur la conception et l'aménagement de l'espace

$10 \mid 2014$

Le paysage à l'épreuve de la transition énergétique

\title{
À la redécouverte d'un paysage hydroélectrique stratifié, entre montagne et plaine
}

Le cas du système Piave - Santa Croce - Val Lapisina dans le Nord-Est de l'Italie

Re-discovering the Strata of a Hydro-electric Landscape - The case of the PiaveSanta Croce - Val Lapisina system in Northeast Italy

\section{Viviana Ferrario et Amelia Maris}

\section{(2) OpenEdition}

\section{Journals}

Édition électronique

URL : http://journals.openedition.org/paysage/11537

DOI : 10.4000/paysage. 11537

ISSN : 1969-6124

Éditeur :

École nationale supérieure du paysage de Versailles-Marseille, Institut national des sciences appliquées Centre Val de Loire - École de la nature et du paysage, École nationale supérieure d'architecture et de paysage de Bordeaux, École nationale supérieure d'architecture et de paysage de Lille, Agrocampus Angers

\section{Référence électronique}

Viviana Ferrario et Amelia Maris, «À la redécouverte d'un paysage hydroélectrique stratifié, entre montagne et plaine », Projets de paysage [En ligne], 10 | 2014, mis en ligne le 31 août 2014, consulté le 15 octobre 2020. URL : http://journals.openedition.org/paysage/11537 ; DOI : https://doi.org/ 10.4000/paysage. 11537

Ce document a été généré automatiquement le 15 octobre 2020

Projets de paysage 


\section{À la redécouverte d'un paysage hydroélectrique stratifié, entre montagne et plaine}

Le cas du système Piave - Santa Croce - Val Lapisina dans le Nord-Est de l'Italie

Re-discovering the Strata of a Hydro-electric Landscape - The case of the PiaveSanta Croce - Val Lapisina system in Northeast Italy

Viviana Ferrario et Amelia Maris

\section{Introduction : paysage de l'énergie}

1 Comme toutes les transitions énergétiques du passé, celle que nous sommes en train de traverser est susceptible de générer une nouvelle configuration territoriale (Smil, 2005). Même si la production, le transport et la consommation d'énergie participent à la construction de l'espace des sociétés et à l'établissement de relations étroites avec les systèmes territoriaux qui les accueillent, les aspects spatiaux de l'énergie sont largement négligés aujourd'hui, avec pour conséquence de nombreux conflits associés au développement des énergies renouvelables. Souvent le paysage est au cœur de ces conflits, en tant qu'objet à préserver d'une altération par les infrastructures nécessaires à l'exploitation "douce » de la ressource énergétique, devenant ainsi luimême un obstacle au développement des énergies renouvelables. Politiques paysagères et politiques environnementales se contredisent ainsi, ou se trouvent au moins condamnées à une laborieuse et insatisfaisante recherche de compromis.

2 Pour dépasser cette impasse, plusieurs chercheurs se sont orientés vers une spatialisation et une territorialisation de la question énergétique (Ghosn, 2010, Puttilli 2014) accompagnées d'une conscience paysagère plus sérieuse dans les politiques énergétiques, et proposant le concept de "paysage de l'énergie " (Nadaï et Van der Horst, 2010). Le but est de faire sortir le paysage d'une appréciation esthétique 
inconsciente des codifications qui l'ordonnent et de lui rendre toute l'épaisseur que lui confèrent la mémoire qu'il porte et le témoignage qu'il délivre sur les relations des sociétés à leurs environnements et à leurs ressources.

3 C'est là l'objectif visé par le projet de recherche "Ressources énergétiques et ressources paysagères » (Briffaud, 2014), sur lequel cet article s'appuie ${ }^{1}$. En construisant une «lecture énergétique » diachronique des paysages montagnards, ce projet vise à faire de ces paysages un support de médiation, rassemblant les acteurs et habitants concernés autour d'un même objet de réflexion et permettant simultanément de penser les connexions entre la question énergétique et de nombreuses autres questions relatives à la gestion des territoires et au développement local. Le paysage " énergétique » - en l'occurrence spécialement celui dont l'exploitation de la ressource hydroélectrique a déterminé les configurations et les dynamiques -, en tant que reflet local et en tant qu'« archive » des relations sociétés/énergie, permet de percevoir les effets territoriaux directs et indirects des choix faits dans le domaine de l'énergie.

Il s'agit ici de développer, dans cet esprit, le cas du Val Lapisina, partie du système hydroélectrique du Piave, dans le Nord-Est de l'Italie. L'intérêt d'étudier cette vallée réside avant tout dans le fait qu'il s'agit du premier grand site d'exploitation hydroélectrique de ce bassin-versant. Son aménagement et réaménagement, à plusieurs reprises au cours du $\mathrm{xx}^{\mathrm{e}}$ siècle, pour l'adapter aux innovations techniques et aux nouvelles stratégies industrielles ou territoriales, traverse toute l'histoire de l'hydroélectricité en Italie : les traces paysagères qu'elle conserve font de cette vallée un cas d'archive société/énergie particulièrement intéressant et complet. En second lieu, le Val Lapisina, autrefois très habitée et siège de nombreuses activités économiques, est aujourd'hui entré dans une phase de déclin démographique et économique très intense, qui pose question au regard de son passé hydroélectrique.

\section{Paysages hydroélectriques}

5 Le développement de l'énergie hydroélectrique en Italie au $\mathrm{xx}^{\mathrm{e}}$ siècle a principalement été étudié sous ses aspects économiques, financiers et industriels (Leonardi, 2004, Reberschak, 2004), sociaux (pour exemple Cafarelli, 2004 sur les vallées de la Carnia ; Pelli, 2004 en Trentin) et finalement architecturaux et paysagers (Fontana, 1981; Selvafolta, 1984 ; Restucci, 1998 ; $c f$. le numéro monographique de Rassegna, 1995). À partir des années 1980, des études dédiées à des systèmes d'implantations spécifiques ou à des territoires singuliers (Jacob et Stahel, 1997; Polatti, 2003) ont contribué à porter vers un plan territorial et paysager un regard auparavant centré sur les ouvrages (centrales, barrages, conduites). Le rapport entre développement hydroélectrique et paysage soulève plusieurs questions et peut être abordé sous différents points de vue, pouvant être résumés comme suit.

- Inscription des infrastructures dans le paysage. Il s'agit d'étudier la "capacité singulière » des infrastructures hydroélectriques « à savoir altérer et en même temps intensifier et dévoiler les caractères originaux du paysage " (Selvafolta, 1998), en produisant de nouveaux paysages. Les " paysages électriques » sont notamment des paysages infrastructurels, créés par les barrages ou par les lignes de transport (Pavia, 1998).

- Impact des infrastructures et conflit avec la protection du paysage. L'impact des infrastructures sur le paysage naturel fut au centre de vives discussions dès le début du développement hydroélectrique à la fin $\mathrm{du}_{\text {xix }}{ }^{\mathrm{e}}$ siècle, et à l'origine de nombreux conflits. Dans le cadre du 
développement de l'hydroélectricité «le paysage a été l'arrière-plan et l'objet d'une profonde opposition... entre le passé et le futur» (Caravaggi, 1998). Ce conflit, perçu principalement comme esthétique et culturel, a pu être engendré, jusqu'à un certain point, par l'impact paysager (ibid.); dans les années 1960, cependant, le conflit a explosé suite à la nouvelle prise de conscience de la complexité des impacts environnementaux de l'hydroélectricité (Fiori, Franzin, Reolon, 2000 et 2009; Sognini, 2006). La compatibilité environnementale des implantations hydroélectriques reste aujourd'hui un problème ouvert, redevenu très actuel du fait du développement de la microhydroélectricité. Producteur par excellence d'énergie renouvelable et, en conséquence, durable par définition, le recours à ce mode de production énergétique soulève, en fait, de graves conflits environnementaux (entre autres Ferrario et Castiglioni, à paraître).

- Patrimonialisation et mise en valeur des infrastructures hydroélectriques. Cinquante ans après la construction des dernières grandes implantations hydroélectriques, le recul nous permet de porter un nouveau regard sur le patrimoine bâti autour des projets hydroélectriques, pour le considérer en tant que patrimoine historique et culturel (Fontana, 1998; Varaschin et Bouvier, 2010 ; Rodriguez, 2012) auquel il est attribué une nouvelle valeur, notamment pour le développement touristique.

- Projet énergétique et projet de territoire. Il s'agit d'un thème dont l'importance a déjà été soulignée, afin de lutter contre la dangereuse «séparation progressive entre les projets d'infrastructures et les politiques territoriales» (Pavia, 1998) et qui a été mis au cœur du projet « Ressources paysagères et ressources énergétiques » (Briffaud et al., 2014).

6 Cette dernière approche nous fait réfléchir à la possibilité qu'un paysage hydroélectrique existant puisse constituer une base pour un nouveau projet de territoire, non pas seulement à travers une mise en valeur du patrimoine industriel, mais grâce à une relecture en profondeur du paysage hydroélectrique lui-même : y retracer les " événements hydroélectriques », comme nous le verrons, est l'occasion de relire le territoire dans ses structures profondes et de réfléchir à un futur fondé sur celles-ci. Les infrastructures développées pour exploiter les ressources ainsi que les paysages effacés et construits par l'exploitation elle-même deviennent à leur tour des ressources.

7 Un projet spécialisé comme le projet hydroélectrique génère parfois des territoires tout autant spécialisés : l'hydroélectricité transforme certaines vallées alpines en couloirs industriels, en créant des «vallées électriques » (Gouy-Gilbert, Dalmasso, Jakob, 2011, p. 15). Du fait de leur force visuelle et de leur caractère imposant dans le paysage, nous imaginons que les infrastructures hydroélectriques sont monolithiques. Toutefois, bien au contraire, les paysages électriques sont souvent très "stratifiés ", là où les implantations ont été réaménagées plusieurs fois (voir Selvafolta, 1998). Ces constatations, portant sur les vallées électriques dela Lombardie, pourraient facilement s'adapter au Val Lapisina. Le développement hydroélectrique du Piave, construit et géré essentiellement par une seule entreprise privée à caractère monopolistique, la Società Adriatica di Elettricità ( $\mathrm{SADE}$ ), a souvent eu un effet sectorialisant. Là où le projet énergétique ne s'intègre pas dans un projet de territoire, ce dernier est alors très fragilisé, lorsque apparaît un changement de ses conditions. C'est le cas de la vallée en question, dont les infrastructures électriques ont accentué considérablement le caractère de couloir infrastructurel. Après l'automatisation des centrales hydroélectriques et la construction de l'autoroute dans les années 1990, la vallée a ainsi fait l'expérience du déclin. 
Figure 1. Val Lapisina : vallée électrique et corridor infrastructurel

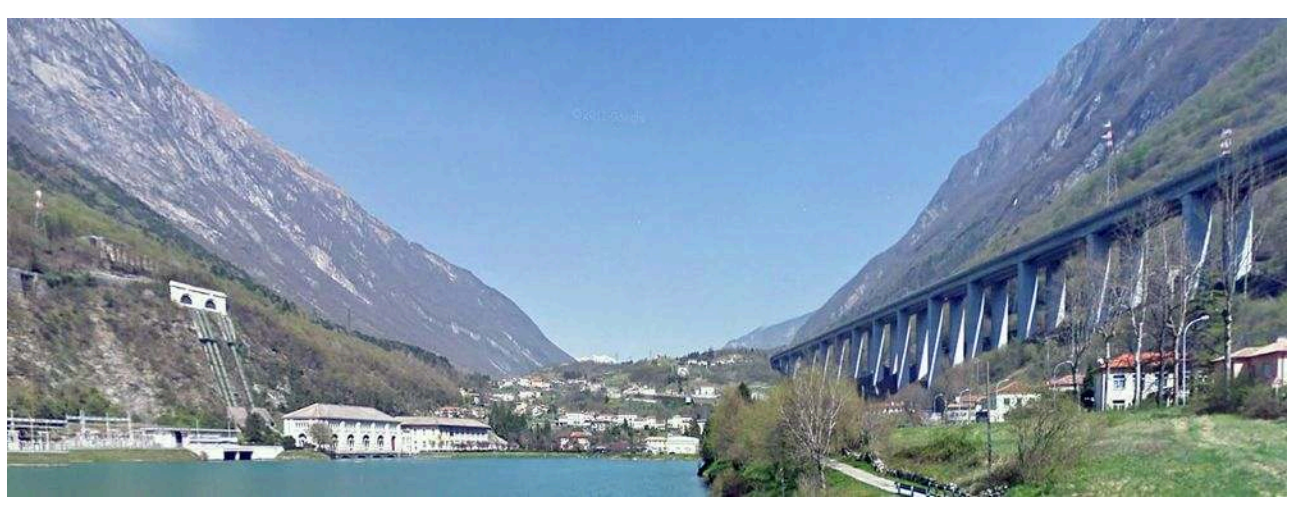

L'actuelle transition énergétique nous invite à relire ce territoire spécialisé : dès lors qu'un cycle de spécialisation est achevé, un nouveau cycle nous permet de repenser le paysage hydroélectrique. Néanmoins, si le nouveau cycle, comme le suggère Fontana en 1998, est celui de la patrimonialisation des infrastructures énergétiques et de leur valorisation touristique, la question de l'intégration au projet de territoire est à nouveau ouverte. Comment intégrer un paysage hydroélectrique très spécialisé, et à quel projet de territoire?

\section{Un paysage hydroélectrique stratifié : le val Lapisina}

Le fleuve Piave traverse les Dolomites orientales et la plaine de la Vénétie pour se jeter dans l'Adriatique. L'exploitation hydroélectrique de son bassin-versant commence au début $d u x^{e}$ siècle pour alimenter en énergie les centres urbains de la plaine et en particulier le pôle chimique de Porto Marghera.

10 L'eau du lac de Santa Croce, un des plus étendus de la région, sera turbinée le long du Val Lapisina, une vallée étroite et accidentée, qui représente l'accès routier naturel à la plaine vénitienne orientale, et celui de la ville de Venise à la montagne. Ce rôle de voie de passage a toujours été confirmé par la politique infrastructurelle gouvernementale : autour de 1830 avec le passage de la grande route d'Alemagna, qui connectait Venise au Tyrol, construite par les Autrichiens qui dominaient la région de 1815 à 1866, et la ligne du chemin de fer, construite entre 1913 et 1926. Peu habitée, la vallée présentait de modestes ressources agricoles et pastorales, du fait de sa morphologie accidentée. Elle est décrite par les voyageurs du XIX ${ }^{e}$ siècle comme un lieu enfoncé et étroit, solitaire et spectral (Edwards, 1872), auquel on accède par la gorge rocheuse de Serravalle, un lieu qui n'est pas mentionné dans les guides, sinon pour l'horrible légende du lac Morto qui faisait se noyer dans ses eaux fatales tous les nageurs (Gilbert, 1864).

La complexité géomorphologique du Val Lapisina est due à l'ancien parcours d'une des deux branches du glacier du Piave, qui est descendu des Dolomites pendant le Quaternaire, et à des éboulements, en particulier celui du Fadalto, qui a créé le lac de Santa Croce. Les autres trois lacs, disposés le long de la vallée à différentes altitudes, sont attribuables à l'action du glacier : le lac Morto, à 274 mètres d'altitude, l'étang de Restello à 177 mètres d'altitude et, enfin, le petit lac de Negrisiola, à 160 mètres.

12 Les petits lacs formant un système naturel de bassins d'accumulation des eaux et la proximité avec la plaine en cours d'industrialisation à la fin $\mathrm{du} \mathrm{XIX}^{\mathrm{e}}$ siècle, où 
commence à se développer une importante demande en énergie, rendent ce site extrêmement propice à l'exploitation hydroélectrique. Entre les dernières années du $\mathrm{XIX}^{\mathrm{e}}$ siècle et les premières années du $\mathrm{XX}^{\mathrm{e}}$ siècle, différents projets d'exploitation du site sont présentés à l'autorité des eaux, jusqu'à ce que, en 1911, la première autorisation arrive et le Val Lapisina commence à être transformée en un véritable paysage électrique ${ }^{2}$.

Dans les pages qui suivent nous nous appuierons sur la reconstitution du "scénario paysager de l'énergie" du Val Lapisina tout au long du $x^{e}$ siècle. Un "scénario paysager de l'énergie » permet de "rendre compte des interactions qui existent entre les évolutions paysagères, les différents modes d'exploitation de la ressource énergétique et les autres formes d'exploitation des territoires montagnards (agricoles, forestières et touristiques) » (Briffaud et al., 2014).

Il s'agira de comprendre ici comment les événements s'inscrivent dans le paysage et comment ils sont eux-mêmes influencés par le paysage. Dans le texte, les événements se déroulent en reconstruisant la trame diachronique du paysage hydroélectrique de la vallée dans son contexte régional. À côté du texte, pour élargir et approfondir la compréhension spatiale et territoriale du paysage hydroélectrique de la vallée, sont décrits, dans des encadrés, les paysages électriques eux-mêmes, leurs interactions avec les autres paysages (agricoles, forestiers, touristiques, etc.), leurs « annexes » proches et éloignées, leur traces persistantes encore distinguables aujourd'hui.

\section{Lecture diachronique du paysage hydroélectrique du Val Lapisina : les événements et leurs traces visibles et invisibles}

\section{Début de l'exploitation hydroélectrique industrielle de la Vénétie}

En Vénétie, l'exploitation hydroélectrique industrielle commence sur l'Adige (1905), sur le Cellina (1905) et sur le Cismon (1909). Toutes les grandes implantations étaient concentrées dans la zone préalpine, à proximité des concentrations industrielles qui allaient se former autour des villes principales. Il s'agissait de centrales au fil de l'eau', qui souffraient dans les périodes d'étiage. C'est pourquoi les carences de la production hydroélectrique était supplées par celles des centrales électrothermiques, alimentées au charbon, construites dans les zones industrielles urbaines (Vérone, Padoue, Venise) (SADE, 1924).

16 Le Piave est le dernier des grands fleuves de la Vénétie à être exploité. Son histoire hydroélectrique remonte à 1899 , quand les ingénieurs Colle et Milani présentent un projet pour dériver ses eaux à Ponte nelle Alpi, les amener dans le lac de Santa Croce et les utiliser le long du Val Lapisina. L'électricité produite aurait dû servir pour l'électrification de la ligne de chemin de fer «Conegliano - Vittorio Veneto - Ponte nelle Alpi » - dont le premier tronçon avait été terminé à Conegliano en 1879 et devait être prolongé jusqu'en Cadore avec un tramway (Comune di Pieve di Cadore, 1899) c'est-à-dire relier la montagne à la plaine. Mais ce projet est resté irrealisé, jusqu'en 1909, quand la Società Italiana per l'utilizzazione delle Forze Idrauliche del Veneto (dite aussi «Cellina », fondée à Venise en 1900 et déjà active sur le bassin-versant du fleuve Cellina, fournissant l'électricité à la ville de Venise et entrée sous contrôle de la SADE 
en 1907), présente un projet pour l'exploitation hydroélectrique du lac de Santa Croce et des chutes et petits lacs naturels qui se suivent dans le Val Lapisina. Le projet prévoyait de dévier $6 \mathrm{~m}^{3} / \mathrm{s}$ d'eau du fleuve Piave pour les importer vers le lac de Santa Croce. Les autorités, préoccupées par les effets sur le Piave et peut-être du fait d'oppositions locales, concèdent en 1911 à la société Cellina la possibilité d'utiliser pour l'instant seulement $3 \mathrm{~m}^{3} / \mathrm{s}$ de l'eau provenant du lac de Santa Croce, pour les turbiner dans les deux centrales qui seront construites dans la vallée. Même si le projet sera achevé seulement dans les années 1930, le Val Lapisina change beaucoup et très rapidement (encadré 1 ).

Encadré 1. Exploiter des chutes et des lacs naturels (1899-1918)

L'exploitation hydroélectrique du Val Lapisina commence au début du $\mathrm{xx}^{\mathrm{e}}$ siècle. Le paysage de la vallée change radicalement. L'eau du lac de Santa Croce est convoyée dans une galerie forcée, longue de plus de deux kilomètres, creusée dans la roche, recouverte de béton, et traversant le col de Fadalto jusqu'au bassin de mise en charge. De là, deux conduites forcées en plein air, longues de $160 \mathrm{~m}$ avec un diamètre de $1,80 \mathrm{~m}$, portent l'eau jusqu'à la centrale de Fadalto, où elle est turbinée et déchargée dans le lac Morto. Avec la construction de la centrale, le petit marais sur les bords du lac en amont est assaini. Le niveau du lac en aval est contrôlé par un petit barrage et par une écluse, d'où les eaux empruntent un canal artificiel, à ciel ouvert, pour être amenées à un deuxième bassin de mise en charge. De là, deux conduites forcées de $450 \mathrm{~m}$ de longueur les amènent à une deuxième centrale, au bord du lac de Nove.

Ces deux conduites, avec leurs centrales, les lignes électriques et l'aménagement des bords des lacs ont marqué le paysage du Val Lapisina comme paysage hydroélectrique stricto sensu. . Certains éléments appartenant à cette phase ont été perdus, comme l'ancienne centrale de Fadalto (1914), remplacée par un autre bâtiment dans les années 1960 . De cette ancienne installation, restent aujourd'hui, autour du lac Morto, la « glissière » en dessous des conduites forcées et la « porte » , en amont, d'où les conduites sortaient de la roche. Plus bas, vers Nove, les conduites forcées de la deuxième chute ont été retirées, mais la façade du bassin de charge montre encore une agréable arcade très panoramique. De même, la centrale de Nove (1913) existe encore, même si elle n'est plus utilisée que comme dépôt. 


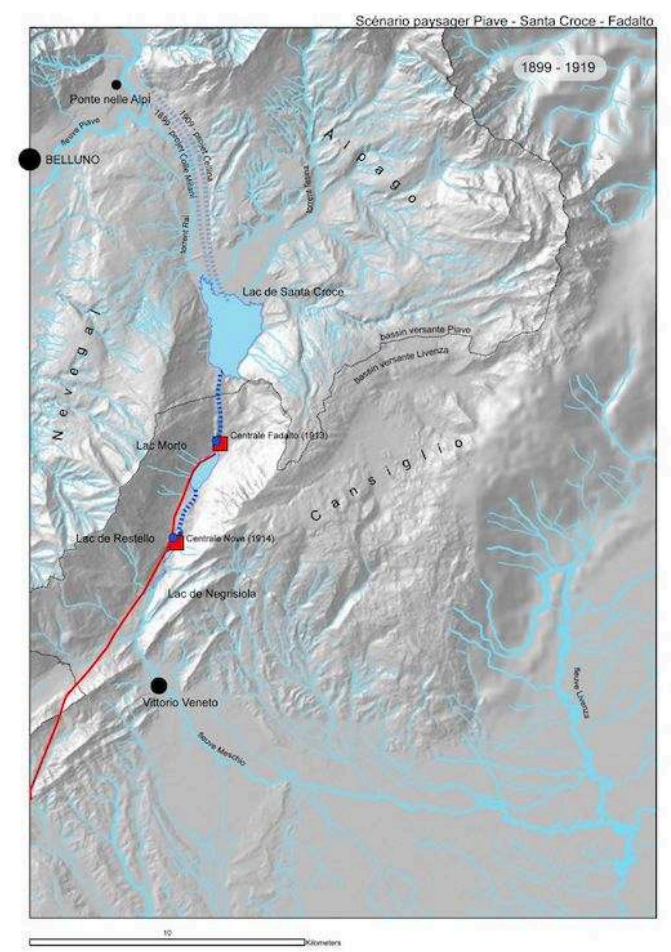

Exploiter des chutes et des lacs naturels (1899-1918).

\section{Du régime fasciste à la nationalisation de l'énergie électrique}

Tout de suite après la Première Guerre mondiale, alors que le prix du charbon augmente sérieusement et qu'au même moment la SADE renforce sa politique de monopole, il devient indispensable de doter le système de production électrique de la Vénétie d'une grande réserve saisonnière : le lac de Santa Croce devient alors un bassin artificiel destiné à contenir les eaux du Piave. La dérivation du Piave est importante, car elle ajoute aux eaux turbinées du Val Lapisina, qui peut compter sur un bassin (l'Alpago) de seulement $150 \mathrm{~km}^{2}$, l'immense bassin-versant du Piave, de presque 1700 $\mathrm{km}^{2}$ (SADE, 1924).

En 1911, pour la dérivation des eaux du Piave, les autorités avaient demandé un complément d'étude sur le régime hydraulique du fleuve, qui devait être réalisé dans les années suivantes. Pour convaincre les autorités, il fallait avoir non pas seulement des raisons strictement techniques, mais aussi des arguments à même d'influencer l'opinion publique: la «naturalité » du nouveau parcours des eaux du Piave ${ }^{4}$, la possibilité d'assainir les marécages de Palù (encadré 2 ), les besoins de la plaine en eau et en énergie pour l'industrialisation et pour l'agriculture (encadré 3).

Encadré 2. Le paysage perdu des marais montagnards (1919-1962)

Apres la Première Guerre mondiale, la société Cellina obtient le permis de dériver du Piave jusqu'à $30 \mathrm{~m} 3 / \mathrm{s}$ et de surélever le niveau du lac de Santa Croce. Entre le fleuve et le lac s'étendait une zone marécageuse causée par la faible différence de 
niveau, le Palù. Elle était traversée par le canal Rai, construit par la République de Venise pour transporter vers le Piave et jusqu'à Venise le bois provenant de la forêt « bandita » du Cansiglio. La zone était classée dès 1885 parmi les marais à drainer, et l'idée est d'utiliser le Rai comme collecteur des eaux. Mais on attendait de résoudre le problème de la « sistemazione montana » (l'aménagement de la montagne) forestière et hydrogéologique du bassin-versant du Rai, réalisée dans les années 1920, de façon que le Rai lui-même ne puisse pas être rempli par les matériaux solides transportés par l'eau (Inea, 1942). Le projet de surélever le niveau du lac de Santa Croce permit de réduire de moitié la surface marécageuse, en l'inondant avec les eaux prises dans le Piave. Du marécage de Palù il ne reste aujourd'hui que le toponyme.

Figure. 3. Les eaux du Piave dans le lac de Santa Croce (1919-1962)

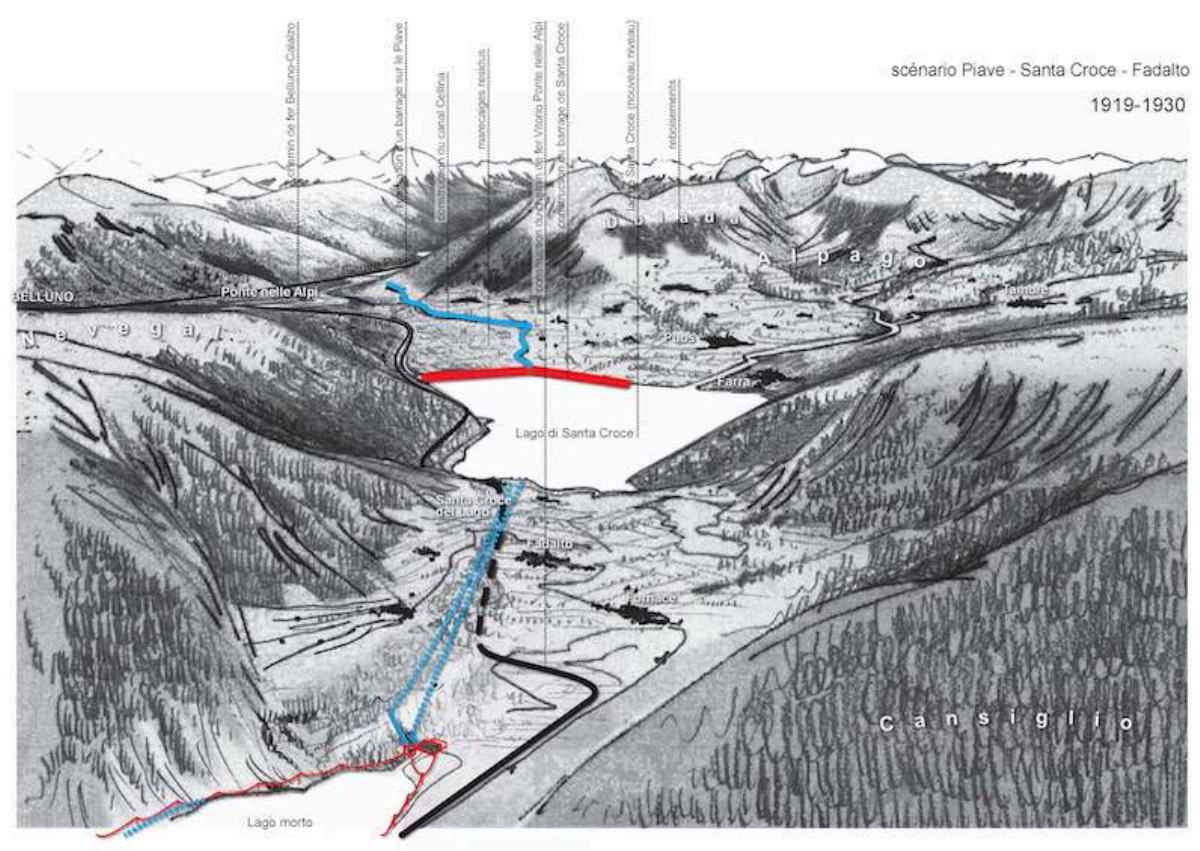

Le paysage hydroélectrique du Val Lapisina a non seulement son reflet dans les villes mais dans l'ensemble de la plaine vénitienne, jusqu'à la mer. En 1919, 70 km plus au sud, le tout nouveau pôle chimique de Porto Marghera, futur cœur industriel de «la région italienne qui avait le plus souffert de la guerre, moralement et matériellement " (SADE, 1924, p. 43) avait été établi par Antonio Volpi, patron de la SADE, et était prêt à consommer des millions de KWh. En 1919 déjà, la société Cellina commence à construire une première prise provisoire à Soverzene, ainsi que le canal artificiel, une partie couverte et une partie à ciel ouvert, destiné à conduire l'eau du Piave jusqu'à Santa Croce. Au même moment, l'ingénieur Vincenzo Ferniani, qui avait déjà conçu les centrales de Fadalto et Nove, est chargé d'un nouveau projet pour augmenter la dérivation à $30 \mathrm{~m}^{3} / \mathrm{s}$, afin de porter le niveau du lac à la hauteur maximale de $386 \mathrm{~m}$. L'autorisation est obtenue en 1922. Les travaux continuent jusqu'en 1930, avec la mise en service de la centrale de Caneva. Ils font de ce système l'un des premiers en Europe, avec une puissance totale de $200000 \mathrm{~kW}$ et une production de 800000 millions de $\mathrm{kWh}$ (SADE, 1924). 
Encadré 3. Les « annexes » de la vallée électrique en plaine : énergie, irrigation, drainage

Le système Piave-Santa Croce a été pensé pour exploiter au maximum la marge septentrionale de la région de Vénétie, pour fournir en énergie les centres industriels de la plaine. Mais il a aussi été pensé pour valoriser l'interface sud du système, devenue tout aussi importante puisqu'il met en relation directe la production hydroélectrique et les usages agricoles, très importants pour le développement de la région Vénétie et en même temps essentiels dans la logique des politiques autarciques du régime fasciste.

En premier lieu, il s'agissait de garantir l'irrigation de la haute plaine sèche entre le Piave et le Livenza. Dans cette campagne difficile à irriguer, du fait de son niveau très haut par rapport au Piave et de son régime torrentiel, la sècheresse faisait souvent perdre les récoltes. Un stockage au niveau du lac de Santa Croce permettait de prendre l'eau pour l'irrigation dans un lieu bien plus élevé. La SADE établit de nouvelles alliances : un consortium pour l'irrigation de la plaine entre le Piave et le Livenza est constitué grâce à l'accord pour la restitution de l'eau du Piave à Vittorio Veneto, après l'avoir turbinée dans la centrale de Castelletto, construite 50 mètres en amont de la dernière centrale de Caneva, notamment pour répondre aux besoins d'irrigation.

En second lieu, il s'agit de contribuer à l'assainissement et à la mise en culture des zones littorales marécageuses de la plaine de la Vénétie, à l'époque objets des politiques publiques de « bonifica ». Afin de drainer des terrains souvent situés audessous du niveau de la mer, il faut pomper, ce qui nécessite une ressource en énergie. La SADE comprend rapidement l'intérêt de fournir de l'électricité aux consortiums de drainage : leur vendre de l'énergie à bas coûts signifie aider l'agriculture et par conséquent la patrie à atteindre l'autonomie alimentaire et obtenir ainsi un consensus de l'opinion publique sur ses activités. En 1934, l'union des consortiums de «bonifica » du littoral vénitien (Consorzi Riuniti del Basso Piave) conclura un contrat avantageux avec la SADE pour la fourniture d'énergie électrique bon marché.

Les paysages agricoles de la plaine vénitienne sont donc liés au Val Lapisina par un rapport de réciprocité : ils constituent une sorte d'annexe éloigné, mais indispensable pour comprendre son paysage électrique.

Avec ce nouveau projet, les implantations Piave-Santa Croce assument un rôle complètement différent dans le système de production et de distribution de la SADE dans la région Veneto-adriatica. De simple réserve saisonnière durant les périodes d'étiage, le système Piave - Santa Croce devient le cœur et le barycentre du réseau de production et de distribution de la société électrique vénitienne et de la région haute adriatique dans son entier.

Encadré 4. Paysage électrique (1919-1962)

Le Val Lapisina, dans les années 1920, se transforme en un grand chantier. L'essentiel du paysage électrique de la Val Lapisina émerge à cette période : en amont du lac de Santa Croce, la prise sur le fleuve Piave et le canal artificiel qui amène l'eau de la prise de Soverzene jusqu'au lac, sur une longueur totale de 
$10 \mathrm{~km}$ environ. Un barrage en terre, très long mais d'une hauteur modeste (il ressemble plutôt à un grand remblai) est achevé en 1926, donnant sa forme définitive au lac de Santa Croce. En aval du lac de Santa Croce, le long du Val Lapisina, on voit aujourd'hui le bâtiment de la deuxième centrale de Fadalto (1924), avec le bâtiment contenant les appareils électriques à l'arrière. Même si la centrale n'est plus utilisée, et que les conduites ont étés retirées, il reste la double arcade d'où ces dernières sortaient.

Plus bas, sur le lac du Restello, la centrale de Nove (1923-1929), avec la salle des turbines à côté de la salle des appareils électriques, se reflète dans les eaux du lac ; au-dessus, le bassin de charge et le puits piézométrique sortent de la forêt. En aval $\mathrm{du}$ lac du Restello, on peut traverser le pont qui amène au petit barrage et observer d'en haut la centrale de San Floriano (1921-28), où les eaux du Restello étaient turbinées, pour être rejetées dans le lac de Negrisiola. Au bout du dernier lac, une écluse contrôle et sépare l'eau, qui descend dans l'émissaire Meschio, de celle qui va être turbinée dans les centrales successives.

Tout au long de la vallée, il est possible de suivre les traces (tunnels, remblais) de la construction du chemin de fer, qui suivait strictement les implantations hydroélectriques dans les années 1920 et 1930.

Figure 4. L'arcade dont sortaient les conduites de l'ancienne centrale de Nove

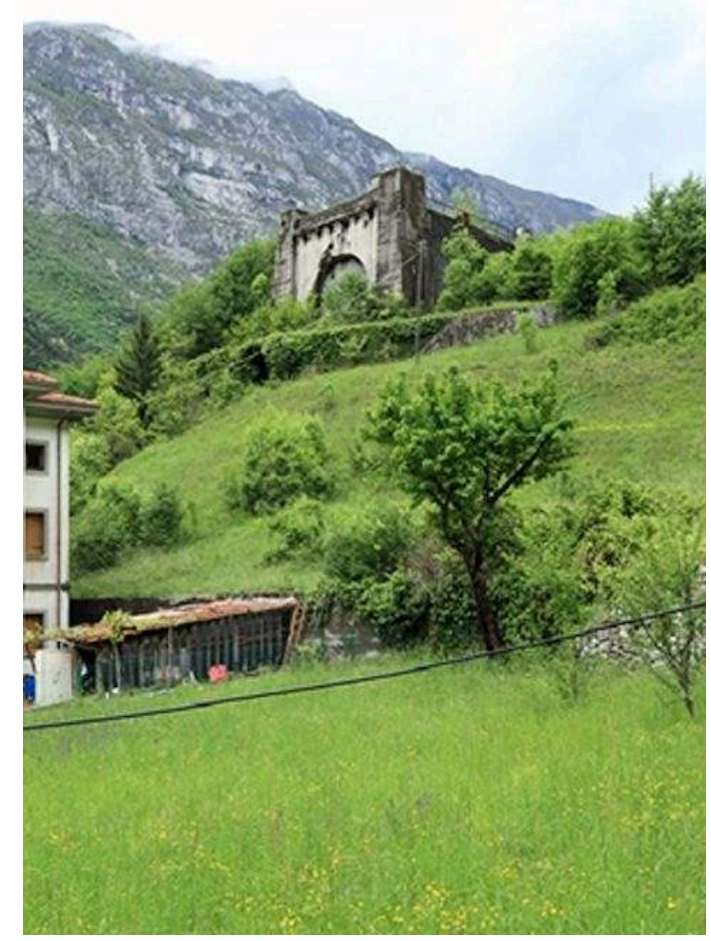

Source : Cristian Guizzo. 


\section{Un paysage électrique à une autre échelle : l'aménagement intégral du Piave}

Entre les années 1930 et les années 1960, la SADE concentre son attention sur le haut bassin du Piave, en poursuivant un rêve destiné à influencer directement non seulement les implantations du lac de Santa Croce, mais toute la gestion du bassinversant du Piave. Ce projet à un nom : "utilisation intégrale» des eaux du Piave, qui, après la Seconde Guerre, sera appelé «Grande Vajont ». L'idée est très simple : intégrer tous les systèmes d'exploitation hydroélectrique d'un bassin-versant, de façon qu'ils fonctionnent ensemble "sans qu'un seul mètre de chute reste inutilisé » (SADE, 1929). Dans les années 1930 et 1940, des dizaines de nouveaux projets de réservoirs commencent à être élaborés dans tout le haut bassin du Piave, jusqu'aux vallées les plus reculées et aux altitudes les plus élevées. Dans cette optique, le paysage hydroélectrique de Santa Croce-Val Lapisina s'étend idéalement vers le nord jusqu'à la nouvelle frontière avec l'Autriche. La Seconde Guerre mondiale ne fera que ralentir le développement: les projets restent dans les tiroirs et les chantiers s'arrêtent, mais pour être repris de plus belle dans les années 1950. Les infrastructures hydroélectriques s'étendent encore au sud et à l'ouest.

Encadré 5. Paysages technologiques extrêmes (1919-1962)

La limite de déviation du Piave à Soverzene ayant été établie sur la base des droits préalables des consortiums d'irrigation, pourquoi donc ne pas s'emparer à cet endroit de toute l'eau, en la restituant au Piave, plus bas, à Nervesa, où elle est nécessaire pour l'irrigation, après l'avoir turbinée dans le Val Lapisina ? Les $25 \mathrm{~km}$ de territoire collinaire entre le Val Lapisina et Nervesa seront traversés par un canal en partie souterrain, le Castelletto-Nervesa. Le projet, qui complète le rêve de l'utilisation intégrale du Piave, est présenté en 1949. Il sera réalisé, partiellement, seulement en 1961. Le canal est encore en fonction aujourd'hui : il représente la ramification infrastructurelle plus étendue vers l'ouest du paysage électrique du Val Lapisina. Les parois du canal sont totalement artificielles même dans le tronçon à ciel ouvert. La naturalité, vraie ou présumée, comme valeur laisse la place à une paysage technologique extrême.

Les kilomètres de tunnels hydroélectriques qui mettent en connexion les différentes implantations déjà établies dans le bassin du Piave après la Seconde Guerre pour réaliser le projet « Grande Vajont » représentent explicitement un défi contre la nature elle-même, qui doit-être amélioré par l'ingénieur hydroélectricien (Semenza, 1950). 


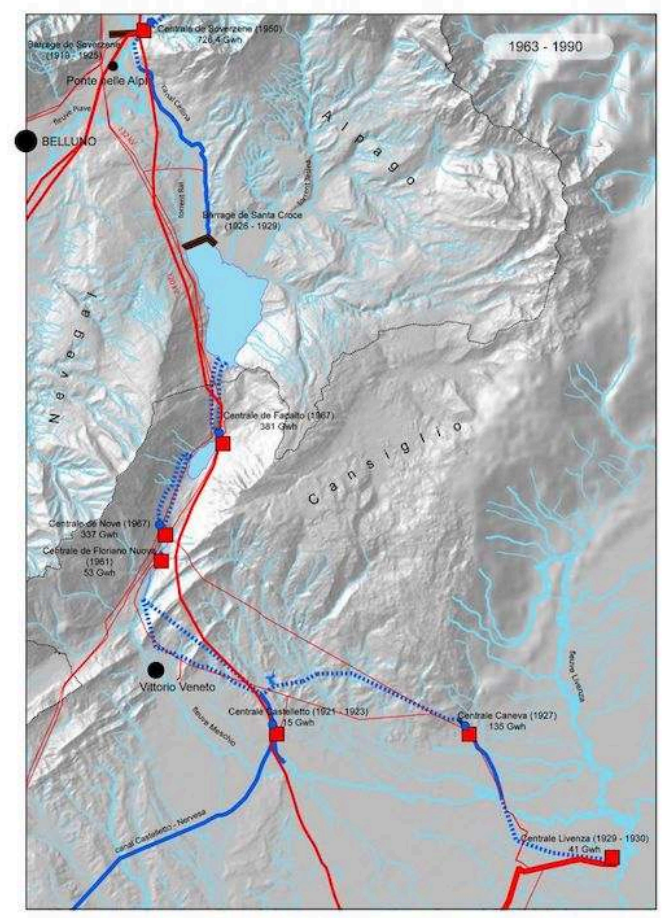

\section{Apres le Vajont}

Le cœur du projet «Grande Vajont » est précisément le réservoir multisaisonnier du Vajont, bâti entre 1959 et 1962 dans une vallée latérale du Piave, près de Longarone, destiné à devenir la «banque de l'eau » (Paolini et Vacis, 1997). L'idée est d'y collecter toute l'eau déjà turbinée dans la partie haute du bassin du Piave, qui le serait ensuite dans la nouvelle centrale de Soverzene, à l'époque l'une des plus puissantes en Europe, avant d'être envoyée au lac de Santa Croce et au Val Lapisina.

Ce grand rêve s'effondrera. Ce qui s'est produit ensuite est connu : le 9 octobre 1963 un gigantesque éboulement de terre (bien prévu mais gravement sous-estimé) tombe dans le lac du barrage du Vajont. L'eau qui sort du lac passe au-dessus du barrage et, en tombant dans la vallée du Piave, raye cinq villages de la carte, parmi lesquels Longarone. On compte près de 2000 morts.

Cette tragédie engendre un profond changement dans la politique énergétique italienne. Après ce désastre, qui a lieu pendant le processus de nationalisation des compagnies électriques, quand toutes les implantations privées passent sous le contrôle de l'Ente Nazionale Energia Elettrica (Enel), la construction de nouveaux barrages s'arrête complètement. Avant 1963 déjà, la dernière phase de développement du réseau hydroélectrique avait dû faire face à un coût marginal croissant, par rapport à la production thermoélectrique. Sous la pression d'une opinion publique désormais absolument opposée aux nouvelles installations, l'Enel décide de renoncer aux projets non réalisés. Entre 1963 et 1969, le projet du Grand Vajont est drastiquement redimensionné. Les projets des grands barrages alpins non encore réalisés sont euxmêmes abandonnés. 
Le Vajont représente aussi une étape pour l'économie de la montagne: en fait, le désastre (et l'arrivée d'argent public pour la reconstruction) fournit l'opportunité d'une politique d'industrialisation de la province de Belluno. De nombreuses zones industrielles sont construites au fond des vallées, à Longarone, dans la vallée de Belluno et près du lac de Santa Croce. Ce qui reste de la zone marécageuse de Paludi accueille la zone industrielle du même nom, une des plus étendue de la province.

\section{Encadré 6. Les paysages « annexes » plus proches : la zone industrielle, la déprise agricole, le « paysage de boue » (1963-1994)}

Apres la tragédie du Vajont, les politiques pour l'industrialisation de la montagne placent une zone industrielle à Paludi, sur ce qui reste du marais assaini avec la construction du lac de Santa Croce. Mais le boom industriel amène les montagnards préalpins à migrer vers la plaine et les activités proto-industrielles qui survivaient dans le Val Lapisina sont abandonnées. La déprise agricole est aussi dramatique : en conséquence, le paysage du Val Lapisina change, se reboise, se referme autour des villages.

Du fait de l'impossibilité d'utiliser le lac artificiel du Vajont rempli par l'éboulement de terre, à partir duquel avaient été comptabilisés les différents droits d'usage de l'eau (jamais recalculés), le bilan est négatif. À certaines périodes de l'année les réservoirs existants ne sont plus suffisants pour satisfaire les droits d'irrigation sans être vidés de façon dramatique. Ainsi, le niveau de l'eau baisse tellement que les rivages boueux des réservoirs sont à découvert. Le phénomène est particulièrement grave pour le lac de Santa Croce. Souvent, pendant la saison touristique, l'agréable paysage hydroélectrique du lac devient un paysage de boue, car en été le besoin en eau pour l'irrigation est souvent à son maximum.

À partir de ce moment, une croissance sans développement est mise en place dans le Val Lapisina. La puissance des centrales et des lignes de transport est augmentée, le rendement du système est amélioré grâce à l'automatisation qui, en revanche, vide les centrales de ses ouvriers (encadré 7).

Encadré 7. Un paysage hydroélectrique qui épaissit, sans s'élargir (1963-1994)

Entre 1967 et 1972, l'Enel, qui ne peut pas étendre son réseau d'implantations hydroélectriques, intervient dans le Val Lapisina en développant les centrales existantes. Le paysage hydroélectrique de le vallée est réaménagé : la première centrale de Fadalto est démolie et une nouvelle centrale moderne est construite à sa place (partiellement souterraine), alors que les conduites du lac à la centrale sont doublées; des pompes sont installées à Fadalto pour remonter les eaux jusqu'à Santa Croce pendant la nuit et afin de les « re-turbiner ». La même chose se passe à Nove : la centrale de 1915 est démolie, celle de 1925 est utilisée comme réserve, une nouvelle centrale est construite sous terre et les conduites sont doublées. A Castelletto, la centrale, en exercice depuis 1923, est agrandie. Audessous des pylônes restent les bâtiments hydroélectriques des années 1960 et 1970, qui ont remplacé les précédentes installations, aujourd'hui perdues ou construites à proximité. Les centrales étant enfouies, seuls les bâtiments de bureaux sont visibles de l'extérieur, comme par exemple à Fadalto. En revanche, la 
ligne électrique à haute tension qui transporte l'énergie vers l'Autriche, d'une part, et vers la plaine, d'autre part, reste très visible.

Enfin, dans les années 1990, l'effet corridor est renforcé par la construction de l'autoroute. Avec un dessin audacieux, deux viaducs traversent longitudinalement le Val Lapisina qui, contourné, perd le peu d'activités liées au passage des touristes présents auparavant : le déclin des activités économiques qui, jusque-là, étaient liées à la fréquentation de la route nationale menant à la montagne, est immédiat. En témoignent les habitations, "locande» et restaurants fermés le long d'une route nationale, aujourd'hui faiblement parcourue et traversant une vallée désormais très peu habitée. Serré entre la montagne et la plaine, comprimé en quelque minute de voyage, son paysage stratifié risque d'être inaccessible.

L'immense viaduc autoroutier sépare définitivement le destin du Val Lapisina de celui du lac de Santa Croce, soudainement rapproché de la plaine : depuis ses riches villes, profitant des vents forts qui soufflent sur le lac, les passionnés de planche à voile montent à Santa Croce même pour quelques heures. Dans les années 1990, le lac de Santa Croce devient le paradis des windsurfers. Il s'agit de touristes non conventionnels, de sportifs, comme ceux qui commencent à fréquenter le lac et le mont Dolada pour faire du parapente.

Par ailleurs, un nouveau développement touristique lié au lac et à la nature a lieu en Alpago. En 2001, la municipalité de Farra d'Alpago lance un concours d'architecture pour l'aménagement de la «plage» du lac de Santa Croce. Un des défis pour les candidats est la résolution du problème du rivage de boue. Le projet lauréat ne sera pas réalisé, mais la plage sera tout simplement aménagée dans la deuxième moitié de la décennie.

Figure 6. Le paysage hydroélectrique stratifié du Val Lapisina aujourd'hui

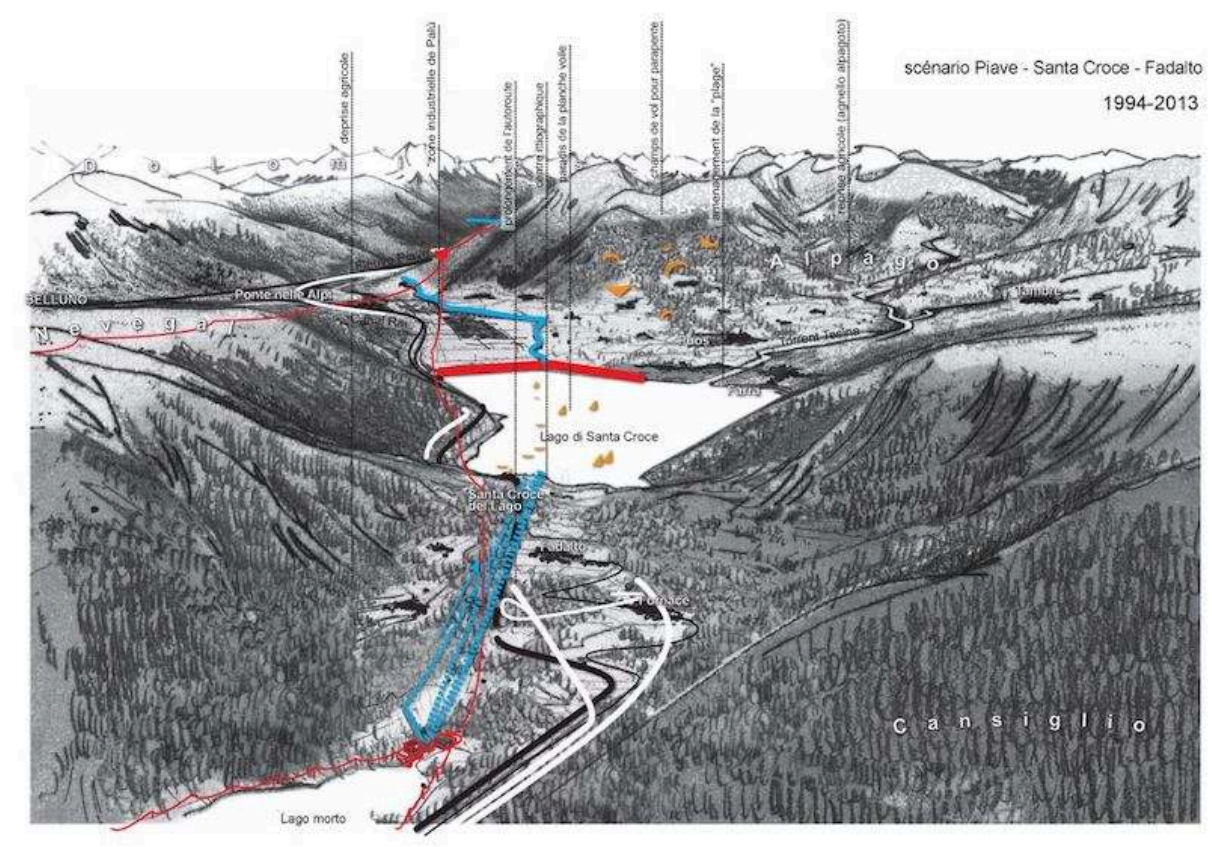

« Scénario paysager » du projet « Ressources paysagères et ressources énergétiques »). 


\section{Stratification paysagère, patrimonialisation et projet de territoire}

30 L'importance de reconnaître et d'étudier la stratification du paysage hydroélectrique à travers la lecture de ses traces a été soulignée par Caravaggi (1998). Sur l'intérêt de cette stratification pourrait se fonder une mise en valeur touristique du patrimoine hydroélectrique. Des indices sont déjà lisibles en Val Lapisina : récemment l'Enel a ouvert certaines centrales «historiques» aux visiteurs et la Centrale de Nove a été visitée par 4000 personnes en 2012.

31 Mais la valorisation des centrales n'est pas suffisante pour infléchir le destin de la vallée. Reconnecter les traces du passé et les indices observés dans un projet intégré de territoire pourrait coordonner dans un cadre commun les initiatives des citoyens locaux ${ }^{5}$, les décisions publiques, les politiques culturelles de l'Enel et l'existence des nombreux visiteurs qui fréquentent déjà la vallée pour leur loisir («plage » du lac Morto, promenades en vélo, pêche sportive).

Pour répondre au déclin social et économique, un projet de territoire capable de prendre en compte tout ce qui est concernant par l'exploitation de la ressource énergétique (l'agriculture, l'élevage, la production d'énergie, l'industrie, le tourisme de proximité) pourrait être aussi utile. Les infrastructures peuvent avoir, dans ce dessin d'ensemble, un rôle déterminant: la voie ferré comme moyen de transport durable pourrait être exploitée en meilleure connexion avec les itinéraires culturels, historiques, et naturels (Maris, 2013); même l'autoroute, qui a entraîné de façon remarquable le déclin de la vallée, peut être considérée comme une formidable voie d'accès.

Désormais les implantations hydroélectriques «appartiennent au paysage» (Val Lapisina Iniziative, 2012). Le moment est venu de délivrer ce territoire de son image de "vallée hydroélectrique » et de "corridor infrastructurel». Une lecture qui permet d'explorer l'épaisseur historique du paysage hérité nous semble un indispensable prémisse pour dépasser cette vision: "Dire ce qui fut à partir de ce qui reste est une manière d'inscrire la houille blanche dans le temps long, celui du passé certes mais aussi celui du présent et du futur. » (Gouy-Gilbert, Dalmasso, Jakob, 2011.)

\section{BIBLIOGRAPHIE}

Briffaud, S. (dir.), « Ressources paysagères et ressources énergétiques dans les montagnes sudeuropéennes. Histoire, comparaison, expérimentation (2012-2014) », rapport de recherche, ADESUMR 5185 du CNRS/Université de Bordeaux Montaigne, Universidad de Granada, Università IUAV di Venezia, Haute École du paysage, d'ingénierie et d'architecture (Hepia) de Genève, Bordeaux 2014. 
Cafarelli, A., « Nascita e primi sviluppi dell'industria elettrica in Carnia » dans Bonoldi, A., Leonardi, A. (dir.), Energia e sviluppo in area alpina : secoli XIX-XX : atti della VII sessione del Seminario Permanente sulla Storia dell'Economia e dell'Imprenditorialità nelle Alpi in Età Moderna e Contemporanea, Milano, Franco Angeli, 2004.

Caravaggi, L., « Natura ed energia. Conflitti e progetti di ricomposizione », dans Pavia, R., Paesaggi elettrici. Territori, architetture, culture, Venezia, Marsilio, 1998.

Dighe di ritenuta degli impianti idroelettrici italiani, vol. 4, Dighe dei Gruppi SADE e Sip, Roma, ANIDEL 1951.

Edwards, A., Untrodden peaks unfrequented valleys, London, Ed. Longman's Green and Co, 1873.

Ferrario, V., Castiglioni, B., « Hydropower exploitation in the Piave river basin (Italian Eastern Alps). A critical reading through landscape ", dans Frolova, M., Prados, M. J., Nadaï A. (dir.), Renewable energies and European landscapes. Lessons from the southern European cases, Berlin, Springer (à paraître).

Fiori, M., Franzin, R., Reolon, S., Il conflitto dell'acqua. Il caso Piave, Sommacampagna (VR), Cierre edizioni, 2000.

Fiori, M., Franzin, R., Reolon, S., Il conflitto dell'acqua. Il caso Piave, atto secondo, Sommacampagna (VR), Cierre edizioni, 2009.

Fontana, V., Il nuovo paesaggio dell'Italia giolittiana, Roma, Laterza, 1981.

Fontana, V., Tra il Veneto e il Friuli ; l'architettura degli impianti idroelettrici, dans Pavia, R., Paesaggi elettrici. Territori, architetture, culture, Venezia, Marsilio, 1998.

Ghosn, R., « Energy as Spatial Project », dans Ghosn, R. (ed.), Landscapes of Energy - New Geographies, $\mathrm{n}^{\circ}$ 2, Harvard University Press, 2010.

Gilbert, J., Churchill, G. C., The Dolomite mountains : excursions through Tyrol, Carinthia, Carniola and Friuli in 1861, 1862 and 1863 : with a geological chapter, London, Longman, Green and Roberts, 1864.

Gouy-Gilbert, C., Dalmasso, A., Jakob, M., Alpes électriques : paysages de la houille blanche, Renage, Dire l'Enterprise, 2011.

Gubler, J., Protasoni, S. (dir.), « Rassegna - problemi di architettura dell'ambiente », Elettricità : Stati Uniti e URSS, Francia e Italia, numéro monographic, $n^{\circ}$ 63, Bologna, Cipia, 1995.

Jacob, M., Stahel, U., (dir.), Girola un'impresa nelle Alpi, Zurigo, Verlag Scheidegger \& Spiess AG, 1997.

Leonardi, A., « Energia sviluppo nell'area trentina e sudtirolese », dans Bonoldi, A., Leonardi, A. (dir.), Energia e sviluppo in area alpina : secoli XIX-XX : atti della VII sessione del Seminario Permanente sulla Storia dell'Economia e dell'Imprenditorialità nelle Alpi in Età Moderna e Contemporanea, Milano, Franco Angeli, 2004.

Mancuso, F., Archeologia industriale nel Veneto, Venezia, Ed. Silvana Editoriale, 1990.

Maris, A.L., « Valorificarea turistica a sistemului hidroelectric Piave - Santa Croce », mémoire de master en géographie du tourisme, universitatea de Vest Timisoara, rapporteur : Ramona Ivan, Corapporteur : Viviana Ferrario, Timisoara, 2013.

Nadaï, A., Van der Horst, D., « Introduction. Landscapes of Energies », Landscape research, $\mathrm{n}^{\circ} 35$, 2010, p. 235-257.

Paolini, M., Vacis, G., Il racconto del Vajont, Milano, Garzanti, 1997. 
Pavia, R., « Sentieri elettrici », dans Pavia, R., Paesaggi elettrici. Territori, architetture, culture, Venezia, Marsilio, 1998.

Pelli, M., Dentro le montagne. Cantieri idroelettrici, condizione operaia e attività sindacale in Trentino negli anni Cinquanta del Novecento, Trento, Fondazione museo storico del Trentino, coll. «Pubblicazioni del Museo storico in Trento. Vesti del ricordo », 2004, vol. 5.

Polatti, F., Centrali idroelettriche in Valtellina : architettura e paesaggio : 1900-1930, Roma, GLF editori Laterza, 2003.

Puttilli, M., Geografia delle fonti rinnovabili. Energia e territorio per un'eco-ristrutturazione della società, Milano, Franco Angeli, 2014.

Reberschack, M., « Dai monti al mare : la SADE e la regione veneto-adriatica » dans Bonoldi, A., Leonardi, A. (dir.), Energia e sviluppo in area alpina : secoli XIX-XX : atti della VII sessione del Seminario Permanente sulla Storia dell'Economia e dell'Imprenditorialità nelle Alpi in Età Moderna e Contemporanea, Milano, Franco Angeli, 2004.

Restucci, « Architetture nuove con rappresentazioni dell'Ottocento », dans Pavia, R., Paesaggi elettrici. Territori, architetture, culture, Venezia, Marsilio, 1998.

Rodriguez, J.-F., « Paysages de l'hydroélectricité et développement touristique dans les Pyrénées », Revue de géographie alpine/Journal of Alpine Research, 100-2, 2012.

SADE (Società Adriatica di Elettricità), Il gruppo Società adriatica di elettricità ed il progresso dell'industria elettrica nella regione veneto-adriatica durante l'ultimo decennio, Venezia, Ed. Stab. tipolit. F. Garzia, 1924.

SADE, Società Adriatica di Elettricità e la sua attività tecnica ed economica dalle origini al 1929, Roma, L’universale Tip. Poliglotta, 1929.

Selvafolta, O., « La costruzione del paesaggio idroelettrico nelle regioni settentrionali », dans Pavia, R., Paesaggi elettrici. Territori, architetture, culture, Venezia, Marsilio, 1998.

Selvafolta, O., « L'immagine del paesaggio tecnologico nella Lombardia del primo Novecento », dans Pirovano, C. (a cura), Lombardia : il territorio, l'ambiente, il paesaggio, Milano, Electa, 1984.

Semenza, C., « Le utilizzazioni idroelettriche ed irrigue del bacino del piave », dans Berti, A., Le Dolomiti orientali. Guida turistico-alpinistica, Milano, Club Alpino Italiano, Touring Club Italiano, 1950.

Semenza, C., « Impianti idroelettrici Piave - Santa Croce », L’Elettrotecnica, vol. X, nº 26, 1923, p. 589.

Smil, V., Energy at the crossroads. Global perspectives and uncertainties, Cambridge (Mass.) ; London, MIT, 2005.

Sognini, G., Acque misteriose : libro bianco sull'uso delle acque nei grandi impianti idroelettrici in provincia di Sondrio, Sondrio, Cooperativa editoriale quaderni Valtellinesi, 2006.

Teti, V., Storia dell'acqua : mondi materiali e universi simbolici, Roma, Ed. Donzelli, 2003.

Tomaselli, C., «Impianto Piave Santa Croce », Le vie d'Italia, vol. XXIX, n ${ }^{\circ}$, Milano, Stab. grafico artistico industriale G. Modiano, 1923, p. 38.

Touring Club Italiano (TCI), Guida pratica ai luoghi di soggiorno e di cura d'Italia - Parte IV la stazioni dei leghi delle Prealpi e degli Appennini, Milano, 1937.

Val Lapisina Iniziative, Da Fadalto a Vittorio Veneto. I laghi blu e la Val Lapisina, film documentaire, Vittorio Veneto, 2012. 
Varaschin D., Bouvier, Y., Le Patrimoine industriel de l'électricité et de l'hydroélectricité, Chambéry, Éditions de l'université de Savoie, 2009.

\section{NOTES}

1. L'article reprend un des «scenarios paysagers » élaborée par l'équipe italienne. Le texte a été rédigé par Viviana Ferrario, avec la collaboration d'Amelia Maris pour les paragraphes 3, 4, et 5 . 2. Les "paysages électriques" (Pavia, 1998) ne se limitent pas aux grands barrages, qui représentent seulement leur partie la plus visible. Chaque implantation hydroélectrique comprend d'autres ouvrages hydrauliques, plus ou moins visibles (conduites souterraines, conduites forcées, canaux, prises) et des ouvrages électriques (centrales de production, centrales de transformation, lignes de transmission). Ces objets et aménagements, apparemment d'impact négligeable par rapport à un barrage, transforment cependant considérablement le paysage. C'est le cas dans le Val Lapisina : un paysage sans barrage, et pourtant profondément marqué par l'hydroélectricité.

3. Sauf pour le Cismon, qui est doté d'un petit barrage en béton, le premier en Italie (Fontana, 1998).

4. L'hypothèse soutenue est que le Val Lapisina était occupée par l'un des deux bras du glacier de la Piave (SADE, 1929) : turbiner les eaux du Piave dans la vallée revenait ainsi à les ramener sur leur ancien parcours naturel.

5. Comme le démontrent les activités de l'association Val Lapisina Iniziative, qui gère depuis quelques années les tous petits lacs à côté de la centrale de Fadalto : cette zone marécageuse aménagée par la société Cellina dans les années 1920 et plus récemment objet d'un reboisement, a été maintenant ouverte au public, et significativement renommée « parco dei laghi blu ».

\section{RÉSUMÉS}

Le développement de l'énergie hydroélectrique tout au long $\mathrm{du} \mathrm{xx}^{\mathrm{e}}$ siècle a souvent transformé les vallées alpines en territoires spécialisés dans la production et le transport de l'énergie. Ces " paysages électriques » se sont quelquefois superposés à d'autres, correspondant à des systèmes différents d'exploitation des ressources - en les remplaçant ou les faisant disparaître. En se spécialisant dans la production énergétique, ces territoires peuvent se trouver fragilisés. C'est le cas du Val Lapisina, transformé au $\mathrm{xx}^{\mathrm{e}}$ siècle en un véritable paysage-corridor infrastructurel et concerné aujourd'hui par un déclin socio-économique significatif. L'article propose une lecture de la stratification de son paysage hydroélectrique et des paysages associés, une meilleure compréhension de ce processus pouvant aider à imaginer un futur différent, où la patrimonialisation et la mise en tourisme des infrastructures hydroélectriques pourraient devenir l'un des aspects d'un nouveau projet de territoire.

The development of hydro-electric energy during the whole of the 20th century often transformed alpine valleys into areas specialised in the generation and distribution of energy. These "electric landscapes" were sometimes layered upon landscapes resulting from other systems for exploiting resources. In other instances they replaced these systems or even caused their disappearance. By specialising in the generation of energy some of these territories became 
vulnerable. This was the case of the Val Lapisina. Literally transformed in the 20th century into an infrastructural corridor-landscape it is now experiencing significant social and economic decline. The article proposes an interpretation of the strata of the area's hydro-electric landscape and of the outlying landscapes. The aim is to provide a better understanding of these landscapes and to make it possible to project a different future in which their assimilation as a heritage and the development of tourism around the hydro-electric infrastructures can provide new development opportunities.

\section{INDEX}

Keywords : stratification of the landscape, hydro-electric landscape, heritage, pre-alpine area Mots-clés : stratification paysagère, paysage hydroélectrique, patrimonialisation, zone préalpine

\section{AUTEURS}

\section{VIVIANA FERRARIO}

Viviana Ferrario est géographe et enseignante chercheuse à l'université IUAV de Venise. Elle a coordonné l'équipe italienne dans la recherche internationale « Ressources paysagères et ressources énergétiques dans les montagnes sud-européennes. Histoire, comparaison, expérimentation ».

viviana.ferrario[at]iuav[dot]it

\section{AMELIA MARIS}

Amelia Maris a un master en géographie du tourisme à l'université de Timisoara, où elle a soutenu sa thèse sur le développement touristique lié à l'hydroélectricité. Entre 2012 et 2014 elle a collaboré à la recherche internationale « Ressources paysagères et ressources énergétiques dans les montagnes sud-européennes. Histoire, comparaison, expérimentation ». maris.amelia.laura[at]gmail[dot]com 